\title{
Design and Implementation of Embedded Driver Fatigue Monitor System
}

\author{
H.M. Shen \\ School of Electromechanical Engineering and Automation \\ Shanghai University, \\ China
}

\begin{abstract}
In order to improve the performance of embedded driver fatigue monitor system, this article designed and implemented a new system based on Soc. This design adopts the ARM as the core processor which is connected to other peripheral by FPGA. The system also uses the embedded Linux to run the main algorithm which consists two Haar-Adaboost classifier to locate the eyes and classify the status of eye by support victor machine. At last by using PERCLOS standard the system will judge whether the driver is fatigue. Experimental results show that the system has a good real-time performance and the processing rate can reach $\mathbf{2 5}$ frames per second.
\end{abstract}

Keywords-fatigue; embedded; PERCLOS; classifier

\section{INTRODUCTION}

Fatigue driving is one of the main reasons for the frequent occurrences of traffic accident today, so the research design and implementation of fatigue driving early warning technology are urgently needed.

Because of the massive calculations of features and limited embedded processor ability, the current realizations on embedded platforms are mainly on DSPs platform. Zhu's detection algorithm is only 3 frames per second on the DSP [1]. Cheng achieved 19 frames per second on DSP platform[2].Although the detection rate at 19 frames per second has entered the threshold of the real-time detection, it is still not enough to prevent fatigue driving.

In this article, a new system is designed and implemented and the speed can reach to 25 frames per second which can timely alert when the driver is fatigue.

\section{SYSTEM DESIGN}

The whole system hardware framework is shown in figure 1 . The APU is the main processing core.850-nm wavelength of infrared camera is used to obtain face images. The FPGA is used to drive AD7511 to display. High-speed SD memory card is used to store the embedded system and FPGA configuration files. The whole system uses DDR3.

\author{
M.H. Xu \\ School of Electromechanical Engineering and Automation \\ Shanghai University, \\ China
}

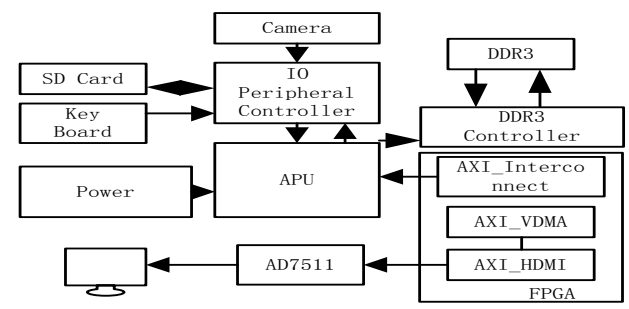

FIGURE I. THE HARDWARE DESIGN OF DRIVER FATIGUE MONITOR SYSTEM

The process of the Fatigue driving warning system is shown in figure 2. The whole system can be divided into four modules: image detection, image process, fatigue detection and real-time display.

The image detection module mainly uses the infrared camera which can reduce the influence of light. Image process module mainly includes image scaling, grayscale conversion and calculation of the integral figure. The fatigue detection module includes face detection, eye detection and fatigue judging. The real-time display module displays real-time face images, test results, and fatigue states of alarm information.

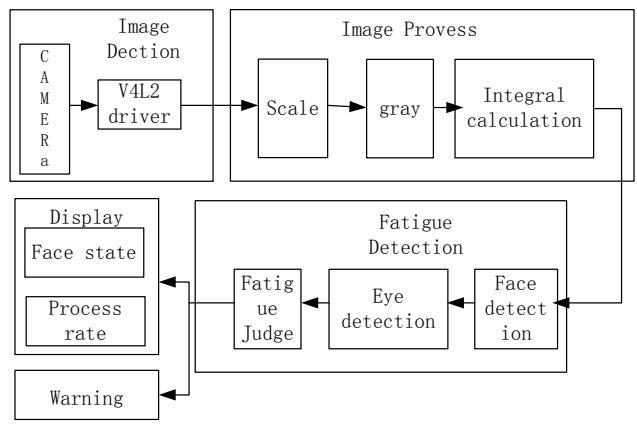

FIGURE II. SYSTEM WORK FLOW

\section{THE REALIZATION OF THE ALGORITHM}

\section{A. Image Capture}

Because of the great influence of environmental light, especially in the day, normal camera is unable to meet the requirements. So the system adopts the infrared camera with the infrared light source. And in order to improve the accuracy, 
infrared human faces and traditional faces should be collected and trained.

\section{B. Image Process}

After captured the infrared image, the image will be reduced and conversed to grayscale. As mentioned in the previous section, the system need to detect human faces and eyes before judging the state of eyes. Viola and Jones [3] proposed a method of fast target detection framework which is one of the best methods, so this article adopts their framework. In the framework the integral figure should be calculated. Integral figure is in order to accelerate the Haar feature extraction. And the equation is

$$
I(x, y)=\sum_{y \leq x} x i(x, y)
$$

After the traversal of integral graph, a rectangular area on the grey value can be calculated by the following formula which can be seen as figure 3 .

$$
I(x, y)=I(x, y)+I(x-1, y)-I(x, y-1)-I(x-1, y-
$$

1)
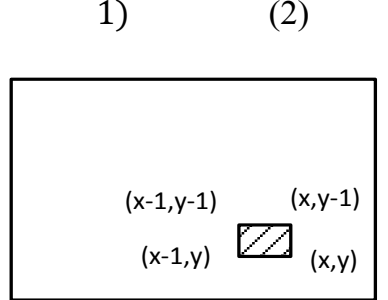

FIGURE III.

THE EXAMPLE OF INTEGRAL CALCULATION

\section{Face Location}

After the calculation of the integral figure, haar features will be imported to face Adaboost classifier. The Adaboost cascade classifier is composed of a series of weak classifiers. And most of the area can be ruled out after previous levels of weak classifiers which is shown in figure 4.

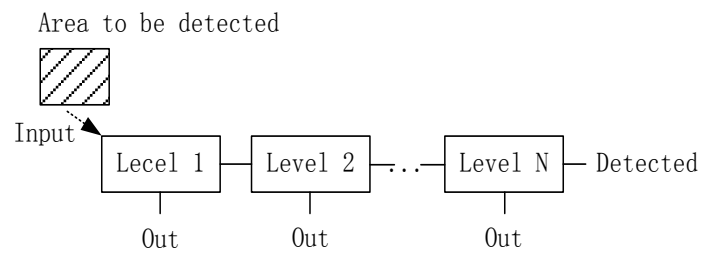

FIGURE IV. $\quad$ ADABOOST CLASSIFIER

The target's coordinates will be return once the target has been successfully classified.

\section{Eye Location}

After located the human face, eyes will also be located by Haar-Adaboost classifier. In this article, the classifier is trained by 36 x 15 . Similarly, after the eyes has been detected, the image coordinates will be return.

\section{E. The Classification of Eye State}

After the location of eyes, system will analyze the eyes' state by the support vector machine[4] to determine whether the eye meet PERCLOS standard[5].According to the P80 of
PERCLOS standard, the eyes are closed when the eyelids cover $80 \%$ of the pupils. And experiences show that the binary pixels are directly related to the samples' characteristics. Generally, the general linear classifier will be considered at first. And the expression of the liner classifier is as follow.

$$
f(x)=\operatorname{sgn}\left(\sum_{i=1}^{n} \alpha_{i} y_{i}\left(x_{i} \cdot x\right)+b\right)
$$

Due to the nonlinear characteristic, the system adopts the nonlinear classifier which uses the RBF kernel rather than the linear classifier[6][7]. And the expression of the nonlinear classifier is as follow:

$$
\begin{aligned}
& f(x)=\operatorname{sgn}\left(\sum_{i=1}^{n} \alpha_{i} y_{i} K\left(x_{i} \cdot x\right)+b\right) \\
& k\left(x_{i}, x\right)=\exp \left(-\frac{\left\|x_{i}-x\right\|}{\sigma^{2}}\right)
\end{aligned}
$$

Among them, the $\sigma$ takes 0.01 .

\section{F. Fatigue Analysis and Judgment}

According to the standard of P80 of PERCLOS, the equation to judge the driver's fatigue is

$$
\text { PERCLOSE }=\frac{\text { framesofclosedeyes }}{\text { allframes }} \times 100 \% .
$$

Within a certain time $\mathrm{T}$, frames of closed eyes $\mathrm{N}$ make up $80 \%$ or more of the total number of frames in $\mathrm{T}$ will be called fatigue driving which is shown in figure 7.

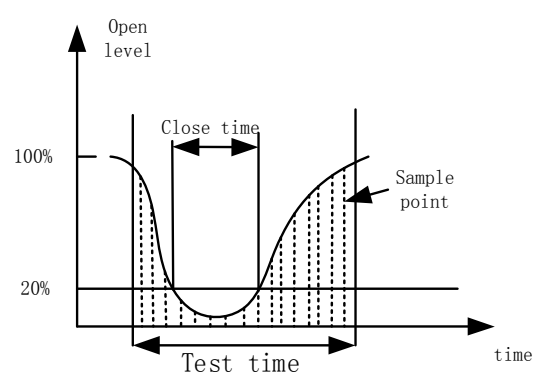

\section{FIGURE V. PERCLOS DIAGRAM}

When the human's body is tired, in the blink of an eye, the time of closed eyes will be more than 1 second. The results show that when detection time $\mathrm{T}$ is stetted to be one second, the frames of closed eyes will be more than 17.And the value of PERCLOS will be greater than $68 \%$ which can be used to judge whether the driver is fatigue.

\section{DESIGN AND IMPLEMENTATION OF THE EMBEDDED SYSTEM}

\section{A. The Design of Soc}

The realization of the system mainly on the platform of Zynq-702 suite. The ARM-A9 is used as the processor. The implementation of system need to use the Soc design software[8] and the configurators of the system which are shown in figure 8 and 9. 


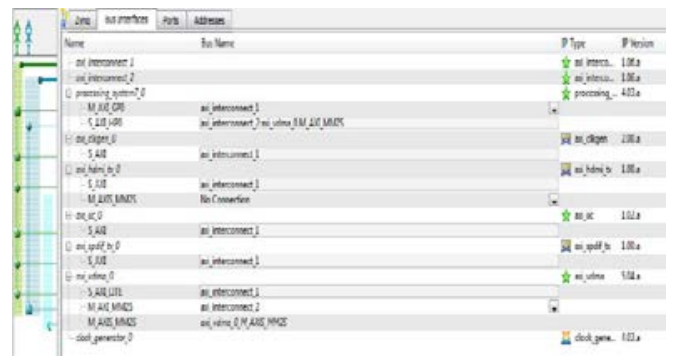

FIGURE VI.

FPGA MODULE CONNECTION DIAGRAM

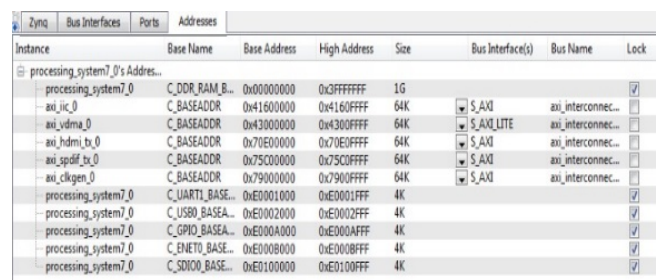

FIGURE VII.

ADDRESS ASSIGNMENT OF EACH MODULE

\section{B. Experiment}

In this article, the embedded system consists of two parts, including Linaro file system and the Linuxkernel [9][10].The hardware is shown in figure 10, which mainly include infrared camera, SD card, power supply and development board. The working system is shown in figure 11.

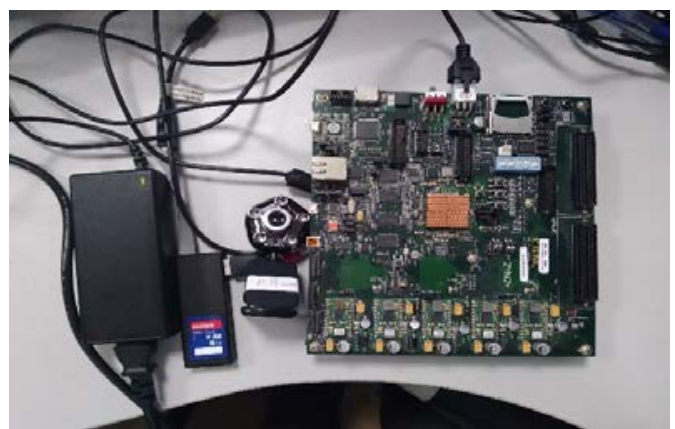

FIGURE VIII.
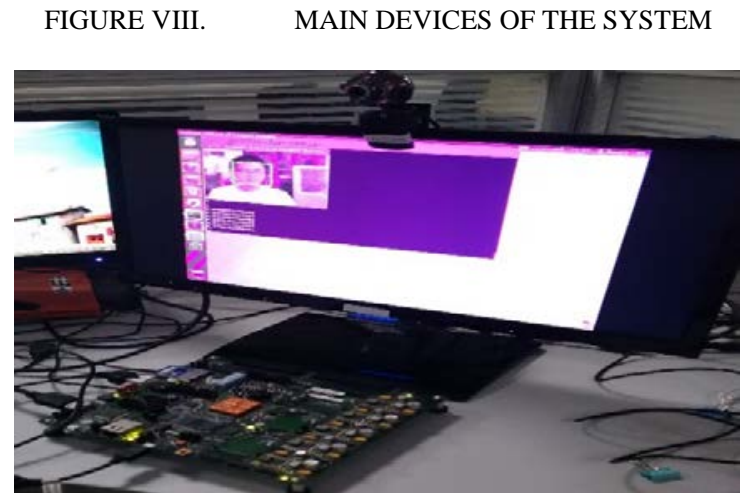

FIGURE IX.

THE WORKING SYSTEM

Through the tests of five volunteers, the experimental results show that in the distance of 0.5 meters to 0.9 meters, this system can run very well. In the night, when the volunteer is tired the alarm information will be displayed which is shown in figure 12.The processing rate on PC can be seen in Figure 13.

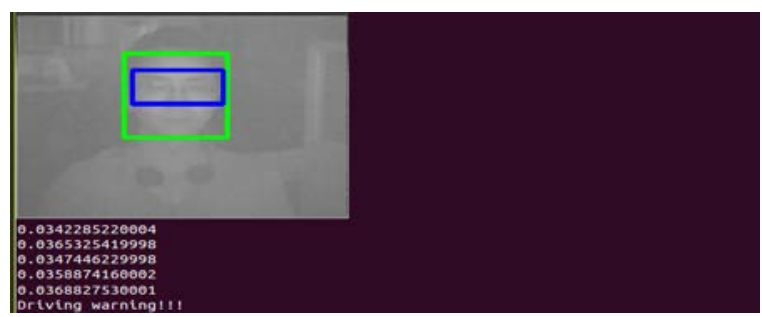

FIGURE $\mathrm{X}$. THE SCREENSHOT OF EMBEDDED PLATFORM

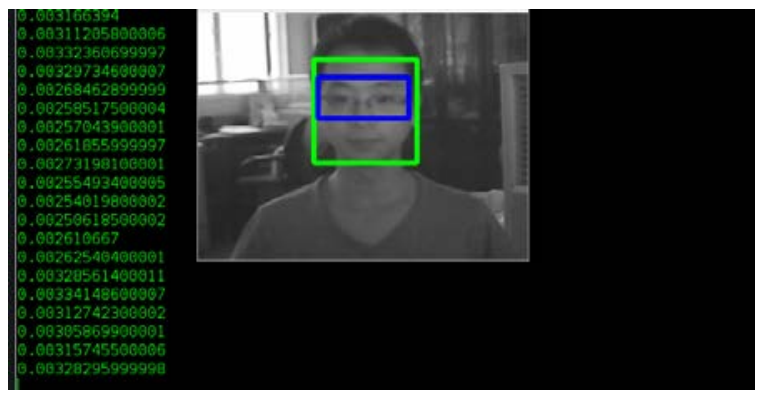

FIGURE XI. SYSTEM TEST ON PC

TABLE I. HE TESTING RESULTS

\begin{tabular}{llll}
\hline tester & $\begin{array}{l}\text { Testing } \\
\text { time }\end{array}$ & $\begin{array}{l}\text { Success } \\
\text { detection }\end{array}$ & accuracy \\
\hline A & $200 \mathrm{~s}$ & $91.6 \%$ & $91.2 \%$ \\
\hline B & $200 \mathrm{~s}$ & $91.3 \%$ & $92.1 \%$ \\
\hline $\mathbf{C}$ & $200 \mathrm{~s}$ & $90.7 \%$ & $90.2 \%$ \\
\hline $\mathbf{D}$ & $200 \mathrm{~s}$ & $92.5 \%$ & $92.6 \%$ \\
\hline $\mathbf{E}$ & $200 \mathrm{~s}$ & $90.1 \%$ & $91 \%$ \\
\hline
\end{tabular}

\section{CONCLUSION}

These results show that this system not is only real-time but also can achieve high precision. And the implementation of this system has great significance for the miniaturization of auxiliary system in the future.

\section{ACKNOWLEDGEMENTS}

This work was financially supported by the financial support of Shanghai Economic and Information Technology Committee by the Annual Projects for Absorption and Innovation of Shanghai Imported Technology under Grant (No.11XI-15), the funded project of the national natural science foundation under Grant (No. 61376028) and the funded project of the Shanghai Science and Technology Commission under Grant (No. 13111104600).

\section{REFERENCE}

[1] CHENG Ruzhong;ZHAO Yong et al. An On-board Embedded Driver Fatigue Warning system Based on Adaboost Method [J]. Acta Scientiarum Naturalism Universitatis Pekinensis.2012,5:719-726.

[2] ZHU Ming,LU Xiaofeng,LU Hengli et al. Transplantation and optimization of AdaBoost face detection algorithm on DSP[J]. CEA, 2014, 50(20): 197-201.

[3] VIOLA P, JONES M. Rapid Object detection usinga boosted cascade of simple features [C] IEEE Conf.Computer Vision and Pattern Recognition, Hawaii: [s.n.],2001: 511-518.

[4] VAPNIK,V.(1995)."Support-vectornetworks".Machine Learning 20 (3): 273.Doi:10.1007/BF00994018. Edit 
[5] DINGES D F, GRACE R. PERCLOS: a validpsychophysiological measure of alertness as assessed by psychomotor vigilance. National Highway TrafficSafety Administration Final Report. Washington, 1998

[6] BERNHARD SCHOLKOPF,ALEXANDER J. SMOLA Learning with Kernels: Support Vector Machines, Regularization, Optimization, and Beyond[M]. USA :MIT Press Cambridge, MA.2001.

[7] OSUNA E, Freund R, GIROSI F. Training support vector machines: An application to face detection[C] IEEE Conf. Computer Vision and Pattern Recognition, PuertoRico: [s.n.], 1997:130-136.

[8] Zynq-7000 All Programmable SoC Software Developers Guide June 19, 2013

[9] Yaghmour K. Building Embedded Linux systems[M].0,Reilly\&ASsociates,hie.2003:60.

[10] MARTIN HINTENNANN.OperatingSystem Components foranEmbeddedLinux System.2007.2 